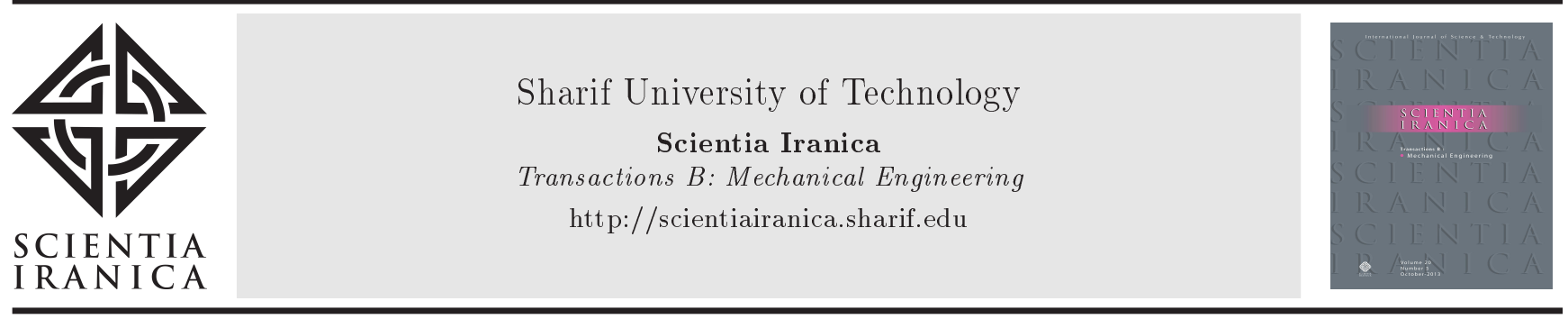

\title{
Intelligent navigation of a self-fabricated biped robot using a regression controller
}

\author{
P.B. Kumar*, C. Sahu, and D.R. Parhi \\ Robotics Laboratory, Department of Mechanical Engineering, National Institute of Technology, Rourkela-769008, Odisha, India.
}

Received 15 December 2017; received in revised form 14 September 2018; accepted 17 November 2018

\author{
KEYWORDS \\ Biped robot; \\ Basic regression; \\ Navigation; \\ Path planning; \\ V-REP.
}

\begin{abstract}
With the growing demand for using biped robots in industrial automation and other related applications, navigation and path planning has emerged as one of the most challenging research topics over the last few decades. In this paper, a novel navigational controller is designed and implemented in a self-fabricated biped robot. After fabricating biped robots equipped with a large set of sensors, a regression controller is implemented on them for the purposes of obstacle avoidance and path optimization. The obstacle distances detected by the biped's sensory network are fed as input parameters to the regression controller, and the output obtained from the controller is the necessary heading angle required to avoid the obstacles present randomly in the environment. The biped is tested in a simulated environment for obstacle avoidance and target-following behavior. Further, to validate the simulation results, a real-time experimental setup is designed under laboratory conditions. The results obtained from both environments are compared in terms of navigational parameters and, then, good agreement is observed between them. Being a relatively new area of research, the navigation of bipeds can pave the way towards industrial automation.
\end{abstract}

(C) 2020 Sharif University of Technology. All rights reserved.

\section{Introduction}

Able to mimic human behavior uniquely and replace human efforts in tedious and repetitive tasks, bipeds have become the center of attraction for many researchers who are engaged in robotic investigation. Bipeds are often preferred over their wheeled counterparts because of their human-like behavior. The use of biped robots has spread to many sectors such as automobiles, manufacturing, industrial automation, medical assistance, aid to elderly people, entertainment source for children, etc. To work freely in an

\footnotetext{
*. Corresponding author. Tel.: +919853934345

E-mail addresses: p.biplabkumar@gmail.com (P.B. Kumar); mechchinu@gmail.com (C.Sahu); dayaldoc@yahoo.com (D.R. Parhi)
}

doi: $10.24200 /$ sci.2018.50018.1466 environment filled with randomly placed obstacles and humans, navigation and path planning remains as the most critical area of investigation. Several researchers have focussed on navigation and path planning of different types of robots over the last few years. Navigation and path planning can be categorized into two parts: model-based path planning and sensor-based path planning. In the model-based path planning, the robot is aware of the source and target positions of travel along with the coordinates of the obstacles present in the environment. In sensor-based path planning, the robot is unaware of any information regarding the obstacle positions, and it has to run a real-time based obstacle detection and avoidance.

One of the major breakthroughs in the area of bipeds is the introduction of Zero Moment Point (ZMP) [1], which has been quite helpful in designing a balancing control for bipeds. $\mathrm{Pa}$ and $\mathrm{Wu}$ [2] designed a hexapod robot with the immediate detection of the 
environment by means of a servo control. Liu et al. [3] proposed a trajectory generation in workspace for adaptive control of biped robots. Sahu et al. [4,5] attempted using nature-inspired techniques in humanoid navigation. Yeon and Park [6] designed a footstep mechanism for abrupt turning and sudden change in the locomotion direction of biped robots. Asa et al. [7] used bifurcation of potential function to discuss the transition between the dynamic behavior of biped and quadruped walking. Iida et al. [8] considered the ideal model of a biped robot using less control and passive elastic joints with an accurate leg design. Rath et al. [9-11] discussed testing a humanoid in simulated and experimental environments using smart algorithms. Rushdi et al. [12] studied the passive gait patterns used in a bipedal walking mechanism. Silva et al. [13] proposed an automated generation of bipedal locomotion without significant knowledge about the task to be performed. Kumar et al. [14-18] developed several intelligent methodologies to ensure the smooth navigation of humanoid robots over complicated terrains. Geng [19] showed the role of passive ankles in the performance of biped robots. They considered the role of the torso and the walking speed of the biped in increasing energy efficiency. Nakamura et al. [20] proposed rhythmic movements controlled by central pattern generators so that biped robots could move stably. Cristiano et al. [21] proposed a mathematical analysis that applies feedback and force sensors to automatically adjust the pattern of a biped robot during inverse kinematics. Parhi et al. [22-24] developed numerous navigational controllers for the hasslefree movement of mobile robotic agents in complex environments. Kajita et al. [25] proposed the motion control of biped robots through auxiliary ZMP. Mirjalili et al. [26] proposed an online-based path planning approach to SURENA III humanoid robot considering various control schemes. Hwang and Ahuja [27] applied a multi-level path planning approach to ensure greater accuracy of results. Atkinson [28] applied basic regression analysis to three specific problems in which least squares estimation and basic regression were varying in nature for predicting the results. He emphasized the correctness of the linear model that represents the regression. Qi et al. [29] modified the potential field method into artificial potential field method for path planning of mobile robots. Asano et al. [30] used disjoint polygons for the construction of any specific design. Bai and Low [31] designed terrain evaluation as a link to body trajectory planning for simplifying the path planning problem. Kala et al. [32] applied two hierarchies, i.e., coarse and finer hierarchies, for the path planning of the mobile robot to overcome random obstacles. Singh et al. [33-35] discussed the application of computational intelligence to ensure smooth and collision-free path generation for wheeled mobile robots. Mohanty et al. [36-38] developed several navigational techniques for path planning of mobile robots using artificial intelligence. They discussed the modification of controlling parameters of basic intelligent algorithms to improve their performance. Sanjuan et al. [39] discussed closed-form solutions for direct and inverse kinematic analysis of a US-RS-RPS 2-DOF parallel robot. Korayem et al. [40] used a robust input shaper for tracking control and vibration reduction of flexible cable-suspended parallel robots.

Although various researchers have focused on the control of biped robots through the use of some algorithms, the subject of path planning and navigation with an obstacle avoidance capability has not been reported significantly. This has motivated the current study to investigate the design of a novel navigational controller that can be used to guide a biped robot through a random set of obstacles present in the environment. According to the extensive survey of the literature, most of the researchers have focused on the navigation of mobile robots. However, a very few studies have reported on the navigation of biped robots. From a real-world application prospective, bipeds are of large significance in assisting human efforts and working as a human replacement. Therefore, bipedal navigation models demand much attention. There is a need for a robust navigational model that can be used in both simulated and experimental environments. Therefore, the current research is aimed at designing a robust navigational controller for path planning of a self-fabricated biped robot. The developed approach has been implemented in a V-REP simulation platform, and the simulation results are verified in an experimental platform developed under laboratory conditions. The results obtained in both of the environments are compared against each other in terms of selected navigational parameters, and they are in close agreement with the minimal percentage of errors.

\section{General overview of regression analysis}

The approach to navigation and path planning problems can be categorized into two types: classical approaches and computational intelligence approaches. Computational intelligence approaches are relatively newer than classical approaches. These two approaches can also be termed as deterministic and nondeterministic ones, respectively. Classical approaches are well known for providing convergent solutions. Since the navigation and path planning of biped robots is new in its own kind, one classical approach called Regression Analysis (RA) is chosen as the method that has been applied in this study for navigational purposes.

Regression analysis is one of the most versatile methods of data forecasting considering the relation- 
ship between dependent and independent variables. By keeping the independent variables fixed in a system, the change in the dependent ones can be analyzed on the basis of independent ones. A general equation of regression can be represented as follows:

$$
\alpha_{i}=a_{1}+a_{2} \beta_{i}+e_{r}
$$

where $\alpha_{i}$ is dependent upon $\beta_{i}$ with $a_{1}$ and $a_{2}$ as the two controlling parameters, and $e_{r}$ accounts for any error. The accumulation of scattered data into a straightline equation is the beauty of regression. Figure 1 represents a basic scheme of regression analysis.

According to Figure 1 , in $x$-axis, some data for independent variables are taken and, in $y$-axis, some data for dependent variables are taken. The straight line represents the accumulation of all the scattered data.

\section{Design of the self-fabricated biped robot}

The current analysis is focussed on the design of a navigational controller for a biped robot. To accomplish the above-mentioned task, a real physical setup of a biped robot has been designed. The designed biped has eight degrees of freedom. After designing the solid model of the biped robot in SOLIDWORKS, it is fabricated by the use of aluminium strips. The biped robot is designed considering the human leg design. Hip geometry of knee and ankle of humans has been considered as important for the design of the biped. In the proposed design of the biped robot, each leg has four degrees of freedom. For each degree of freedom, a servo motor (HiTEC HS-985MG) is used with 5-volt input. The designed biped has two roll rotations in ankle (Right Ankle Roll (RAR) and Left Ankle Roll (LAR)), two pitch rotations in knee (Right Knee Pitch (RKP) and Left Knee Pitch (LKP)), and two yaw and two roll rotations in hip (Right Hip Roll (RHR), Left Hip Roll (LHR), Right Hip Pitch (RHP), and Left Hip Pitch (LHP)). The power unit and the controller are housed in a rectangular channel present in the waist. The synchronized motor rotation is one of the most

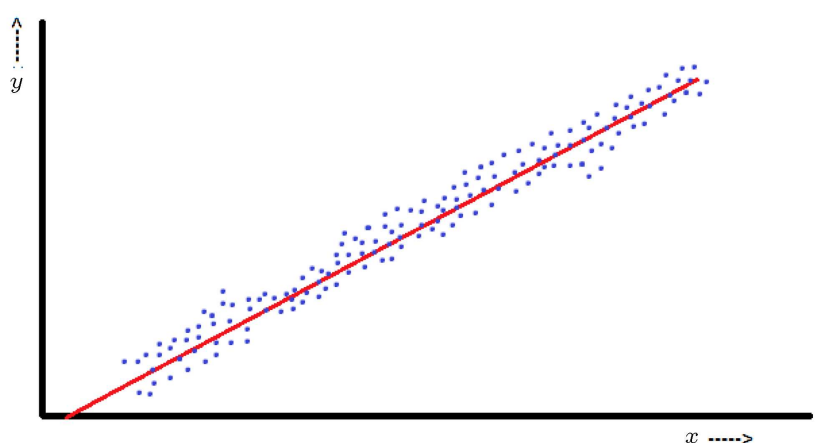

Figure 1. Basic scheme of a regression equation. important aspects of biped locomotion. Therefore, an ARDUINO Mega 2560 controller is implemented on the biped. Two ultrasonic sensors are mounted on the biped for obstacle avoidance purposes. Figure 2 represents the actual view of the biped.

\section{Control architecture for path planning of the biped}

The path planning and navigation problem can be defined as obstacle avoidance and reaching the target position by the shortest path that consumes minimal time. The optimization of the navigation problem is greatly influenced by the navigational parameters that are considered in the optimization function generation. In the navigation of the biped robot, three navigational parameters are considered: Left Obstacle Distance (LOD), Right Obstacle Distance (ROD), and Heading Angle (HA). Out of the three navigational parameters, LOD and ROD act as the input parameters and HA as the output parameter. It can be noted that the two ultrasonic sensors mounted on the fabricated biped robot can account for the LOD and ROD, respectively. HA is the output from the controller, which is generated by the logic implemented on the controller. Figure 3 represents the navigational parameters of the biped robot, used in the current analysis.

The two ultrasonic sensors mounted on the biped record the obstacle distances from the left and right sides as LOD and ROD, respectively, within their range. The HA provides the necessary turning required to reach the target position by avoiding the obstacles.

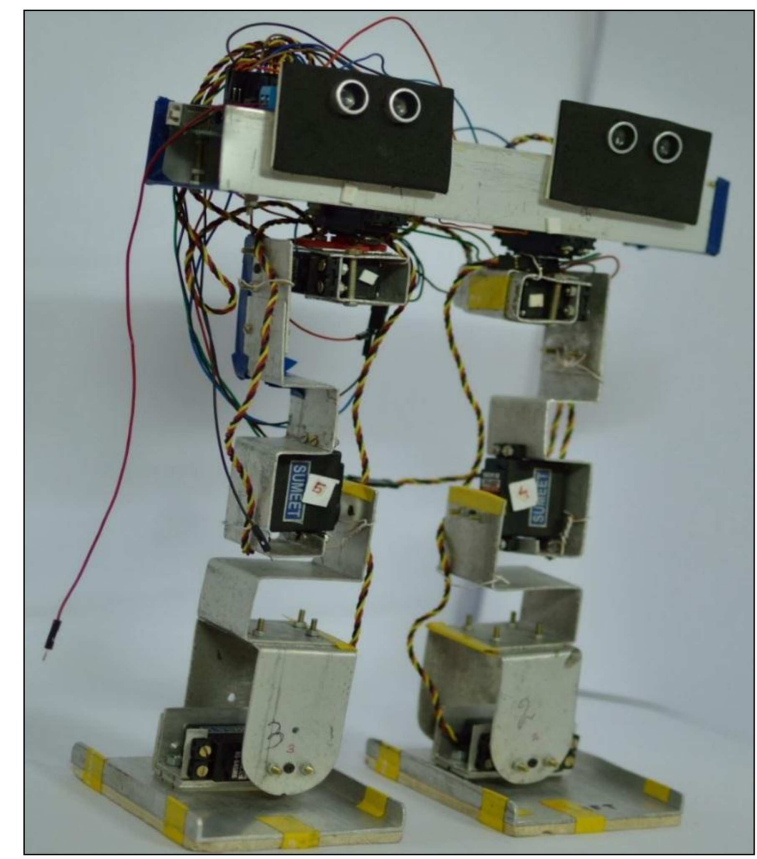

Figure 2. Self-fabricated biped. 


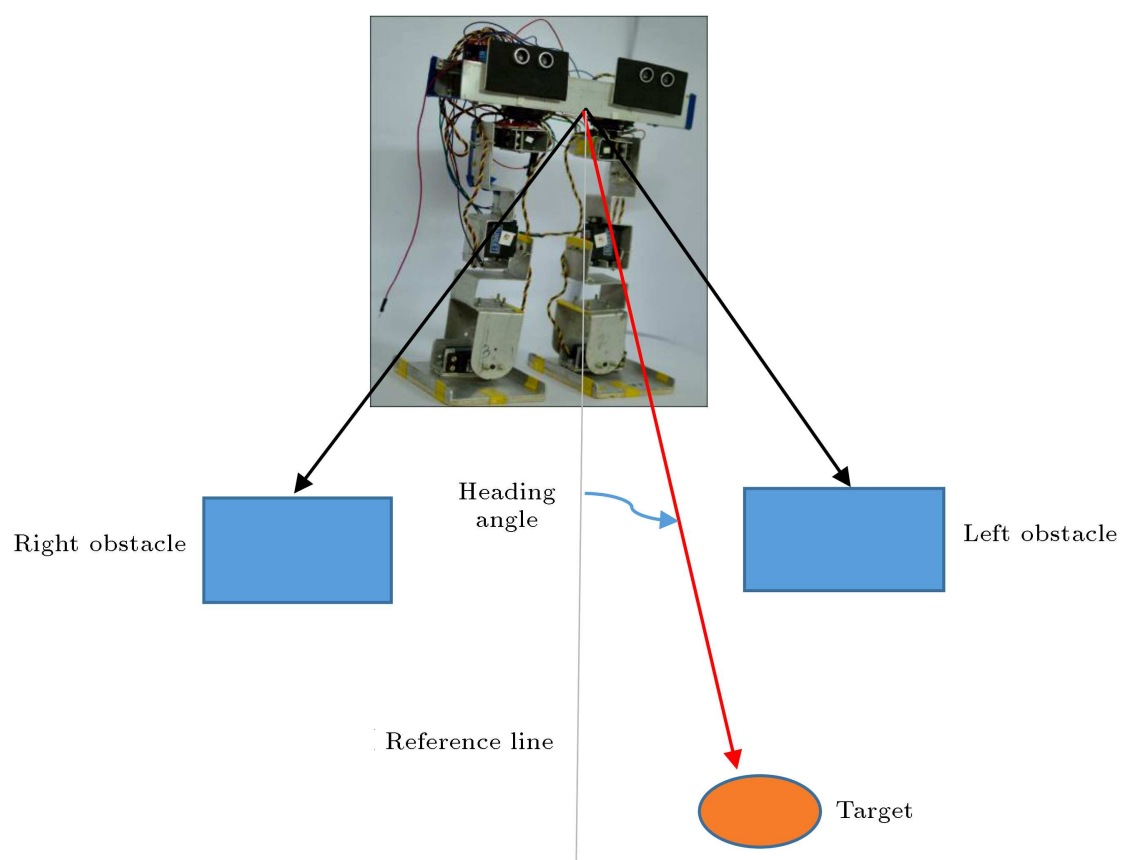

Figure 3. Navigational parameters of the biped robot.

The sensory network of the biped is continuously searching for potential obstacles. Unless the biped detects any obstacle in the environment, it heads towards the target through the target-following behavior. After detecting any obstacle, the regression controller of the biped is activated, and the $\mathrm{HA}$ is decided as per the logic applied to the controller. Based on the environmental conditions, around 750 training pattern data are fed to the controller, depicting random obstacles and source and target positions. Table 1 represents some of the training pattern data used in the current analysis.

The inputs LOD and ROD are represented by centimeters of distance from the biped robot, and the output HA is represented as the angle in degrees. By analyzing one training pattern data shown in Table 1 , the parameters can be understood in detail. The 3rd row data from the training pattern show that $\mathrm{LOD}=$ $30 \mathrm{~cm}$ and $\mathrm{ROD}=70 \mathrm{~cm}$. Accordingly, the right obstacle is far away from the biped, compared to the left obstacle. Therefore, the robot takes a turn at about $20^{\circ}$ towards the right side. It can be observed that some data of HA represent negative values. It is not to be confused with the negative HA that represents some negative heading; rather, it just represents a left turn of the robot. A particular sign convention has been followed in the current analysis. Any rotation to the left side of the reference line is considered as a negative heading angle, and any rotation towards the right of the reference line is considered as a positive heading angle. A zero-heading angle represents no rotation or motion in a straight line following the previous heading angle. After designing the specific sign convention for the bipedal locomotion and the training pattern data, all the training patterns are fed to the regression toolbox of Minitab software. The motive behind using the Minitab software is to formulate a straightline equation from the accumulated scattered data. The equation generated from the Minitab software is presented as follows:

$$
T_{3}=-24.0338-0.006284 T_{1}+0.81356 T_{2},
$$

where $T_{3}=\mathrm{HA}, T_{1}=\mathrm{LOD}, T_{2}=\mathrm{ROD}$.

Based on Eq. (2), the regression navigational controller is implemented on the biped robot. Along with the logic of the regression controller, some reactive behaviors are also implemented on the bipedal locomotion. There are three primary reactive behaviors implemented on the biped robot such as obstacle avoidance behavior, target-following behavior, and wallfollowing behavior. The obstacle avoidance behavior is implemented for avoiding the obstacles present in the environment by generating the required heading angle. The target-following behavior is implemented for heading towards the target in the absence of any obstacles in the environment. The wall-following behavior is a complementary behavior. If there are a series of obstacles existing in the environment and the target is located towards the end of the long barrier, the robot just follows the long barrier without activation of the navigational controller. Figure 4 presents a pseudo code for the regression navigational controller, and Figure 5 presents a general control scheme in a flowchart manner. 
Table 1. Examples of some training pattern data for the navigational controller.

\begin{tabular}{cccccccc}
\hline Serial no. & $\begin{array}{c}\text { LOD } \\
(\mathbf{c m})\end{array}$ & $\begin{array}{c}\text { ROD } \\
(\mathbf{c m})\end{array}$ & $\begin{array}{c}\text { HA } \\
(\mathbf{d e g})\end{array}$ & Serial no. & $\begin{array}{c}\text { LOD } \\
(\mathbf{c m})\end{array}$ & $\begin{array}{c}\text { ROD } \\
(\mathbf{c m})\end{array}$ & $\begin{array}{c}\text { HA } \\
(\mathbf{d e g})\end{array}$ \\
\hline $\mathbf{1}$ & 30 & 50 & $\mathbf{0}$ & $\mathbf{3 1}$ & 60 & 30 & $-\mathbf{1 4}$ \\
$\mathbf{2}$ & 40 & 60 & $\mathbf{1 0}$ & $\mathbf{3 2}$ & 30 & 40 & $\mathbf{1 6}$ \\
$\mathbf{3}$ & 30 & 70 & $\mathbf{2 0}$ & $\mathbf{3 3}$ & 35 & 70 & $\mathbf{1 2}$ \\
$\mathbf{4}$ & 55 & 30 & $\mathbf{- 1 5}$ & $\mathbf{3 4}$ & 30 & 55 & $\mathbf{1 5}$ \\
$\mathbf{5}$ & 30 & 40 & $\mathbf{0}$ & $\mathbf{3 5}$ & 70 & 30 & $-\mathbf{1 7}$ \\
$\mathbf{6}$ & 40 & 30 & $\mathbf{- 5}$ & $\mathbf{3 6}$ & 40 & 40 & $\mathbf{- 2 5}$ \\
$\mathbf{7}$ & 35 & 50 & $\mathbf{2 0}$ & $\mathbf{3 7}$ & 45 & 35 & $-\mathbf{1 8}$ \\
$\mathbf{8}$ & 55 & 40 & $\mathbf{- 1 5}$ & $\mathbf{3 8}$ & 35 & 55 & $\mathbf{2 0}$ \\
$\mathbf{9}$ & 45 & 55 & $\mathbf{0}$ & $\mathbf{3 9}$ & 80 & 45 & $-\mathbf{2 2}$ \\
$\mathbf{1 0}$ & 59 & 0 & $\mathbf{2 0}$ & $\mathbf{4 0}$ & 38 & 59 & $\mathbf{1 8}$ \\
$\mathbf{1 1}$ & 39 & 48 & $\mathbf{1 2}$ & $\mathbf{4 1}$ & 36 & 39 & $\mathbf{1 5}$ \\
$\mathbf{1 2}$ & 46 & 58 & $\mathbf{0}$ & $\mathbf{4 2}$ & 65 & 46 & $-\mathbf{1 1}$ \\
$\mathbf{1 3}$ & 66 & 52 & $\mathbf{- 1 9}$ & $\mathbf{4 3}$ & 46 & 66 & $\mathbf{1 4}$ \\
$\mathbf{1 4}$ & 65 & 33 & $\mathbf{- 1 0}$ & $\mathbf{4 4}$ & 51 & 65 & $-\mathbf{1 2}$ \\
$\mathbf{1 5}$ & 53 & 62 & $\mathbf{1 6}$ & $\mathbf{4 5}$ & 41 & 53 & $\mathbf{1 8}$ \\
$\mathbf{1 6}$ & 61 & 55 & $\mathbf{- 1 8}$ & $\mathbf{4 6}$ & 32 & 61 & $\mathbf{1 6}$ \\
$\mathbf{1 7}$ & 85 & 65 & $\mathbf{0}$ & $\mathbf{4 7}$ & 48 & 85 & $\mathbf{1 2}$ \\
$\mathbf{1 8}$ & 38 & 42 & $\mathbf{2 6}$ & $\mathbf{4 8}$ & 34 & 38 & $\mathbf{1 5}$ \\
$\mathbf{1 9}$ & 42 & 38 & $\mathbf{- 2 5}$ & $\mathbf{4 9}$ & 34 & 42 & $\mathbf{1 6}$ \\
$\mathbf{2 0}$ & 42 & 34 & $\mathbf{- 2 4}$ & $\mathbf{5 0}$ & 0 & 42 & $-\mathbf{1 8}$ \\
$\mathbf{2 1}$ & 55 & 45 & $\mathbf{- 1 8}$ & $\mathbf{5 1}$ & 39 & 55 & $-\mathbf{2 1}$ \\
$\mathbf{2 2}$ & 61 & 71 & $\mathbf{1 4}$ & $\mathbf{5 2}$ & 36 & 61 & $\mathbf{0}$ \\
$\mathbf{2 3}$ & 36 & 38 & $\mathbf{0}$ & $\mathbf{5 3}$ & 53 & 36 & $-\mathbf{1 6}$ \\
$\mathbf{2 4}$ & 48 & 42 & $\mathbf{- 2 0}$ & $\mathbf{5 4}$ & 32 & 48 & $\mathbf{1 8}$ \\
$\mathbf{2 5}$ & 45 & 0 & $\mathbf{1 8}$ & $\mathbf{5 5}$ & 35 & 45 & $\mathbf{2 0}$ \\
$\mathbf{2 6}$ & 53 & 62 & $\mathbf{1 9}$ & $\mathbf{5 6}$ & 45 & 53 & $\mathbf{2 5}$ \\
$\mathbf{2 7}$ & 58 & 36 & $\mathbf{0}$ & $\mathbf{5 7}$ & 70 & 58 & $\mathbf{1 2}$ \\
$\mathbf{2 8}$ & 45 & 48 & $\mathbf{2 2}$ & $\mathbf{5 8}$ & 42 & 45 & $\mathbf{2 8}$ \\
$\mathbf{2 9}$ & 56 & 49 & $\mathbf{- 1 5}$ & $\mathbf{5 9}$ & 45 & 56 & $\mathbf{1 9}$ \\
$\mathbf{3 0}$ & 65 & 20 & $\mathbf{- 1 8}$ & $\mathbf{6 0}$ & 35 & 65 & $-\mathbf{2 6}$ \\
\hline & & & & & & &
\end{tabular}

\section{Navigation of the biped in a cluttered environment}

After formulating the logic of the regression navigational controller and implementing the same in the fabricated biped along with the reactive behaviors, the biped is tested under simulation and experimental platforms. The simulation results are compared with real-time experimental results in terms of navigational parameters.

\subsection{Simulation results for navigation of the biped}

Numerous simulation software products have developed over the last few years to conduct the analysis of bipedal locomotion. However, V-REP has been the center of attraction for bipeds. Compared to other simulation software products, V-REP has certain advantages such as collision detection, better motion planning, and the calculation of the shortest path. $\mathrm{V}$-REP is coded with LUA language based on ANSI $\mathrm{C}$ programming. To analyze the effectiveness of the regression navigational controller, an environment of size $200 \times 200$ units is created in the simulation window of V-REP software. Five static obstacles are placed at random positions. Considering the logic of the regression controller and reactive behaviors, a program has been written in LUA language and implemented on the biped. After specifying the source and target positions, the biped is tested for target-following and obstacle avoidance behaviors. Figure 6 represents the simulation results obtained from the movement of the biped robot using a regression navigational controller. It can be noted that quite a large number of simulation analyses are carried out; however, only a single one has been represented here. 


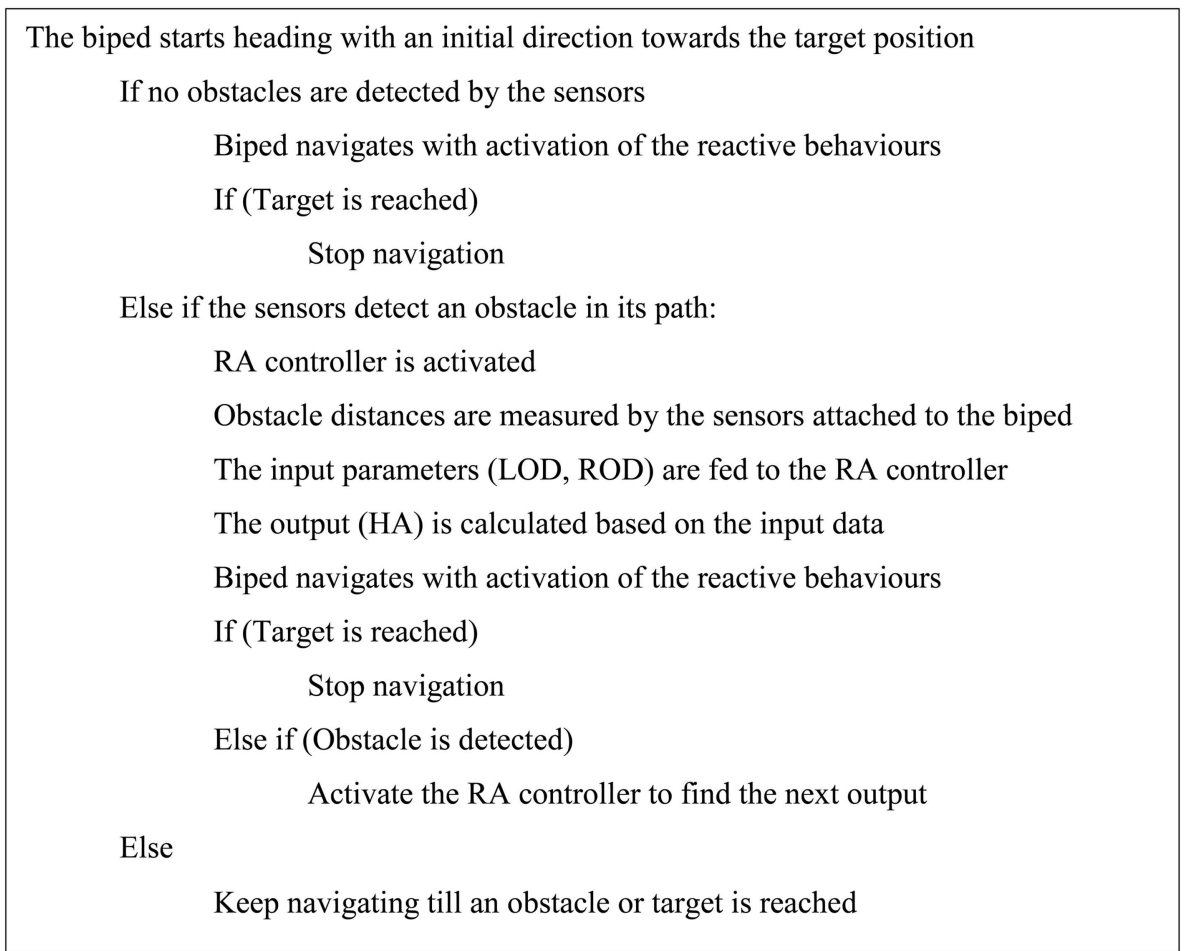

Figure 4. Pseudo code for the proposed RA navigational controller implemented on the biped.

According to Figure 6(a), blue boxes represent the source and target positions, respectively, for the biped robot. Five static obstacles are present at random positions. The path followed by the biped robot shows that it is able to avoid all the obstacles successfully and reach the target position in the shortest path. To compare the results of the simulation analysis with real-time experimentation, path length from the source to the target position and the time spent to cover the path length have been chosen as the two navigational parameters. These two parameters are recorded directly in the simulation window of the $\mathrm{V}$ REP software and noted for further analysis.

\subsection{Experimental results for navigation of the biped}

By preparing a replica of the simulation environment under a laboratory setup, the simulation results can be verified in terms of accuracy. A real-time experimental environment is developed by considering the same size as that of the simulated environment $(200 \times 200$ units). Five static obstacles are located at positions similar to those of the simulation. The logic of the regression controller and the reactive behaviors are applied directly to the controller of the biped by coded language. The biped operates through wired control in the actual environment, and its obstacle avoidance and target-following behaviors are tested in practice. Figure 7 represents the snapshots taken during the actual experiment performed.
According to Figure 7, the environment is kept exactly similar to the simulated environment. The biped is successful in avoiding all the obstacles present in the environment and has also followed a path similar to that of simulation results. Herein, the two navigational parameters, i.e., path length and time consumed, are calculated by means of a measuring tape and stopwatch, respectively, and recorded for further analysis.

\subsection{Comparison of simulation and experimental results}

After performing the navigation of the biped robot in simulated and experimental environments, the results of both environments are compared in terms of navigational parameters. Table 2 shows a comparison of the path lengths, and Table 3 presents a comparison of the time spent between simulated and experimental environments.

According to Tables 2 and 3, the navigational parameters for the experimental environment always provide greater values than those for the simulated environment, because the latter is the ideal environment without any external error factors. Unlike the simulated environment, the experimental environment possesses factors such as loss of data transmission, effects of friction, slippage on the environment floor, etc., accounting for the higher values. The comparison of both results amounts to a maximum error below $6 \%$, which is well inside the permissible limit. The 


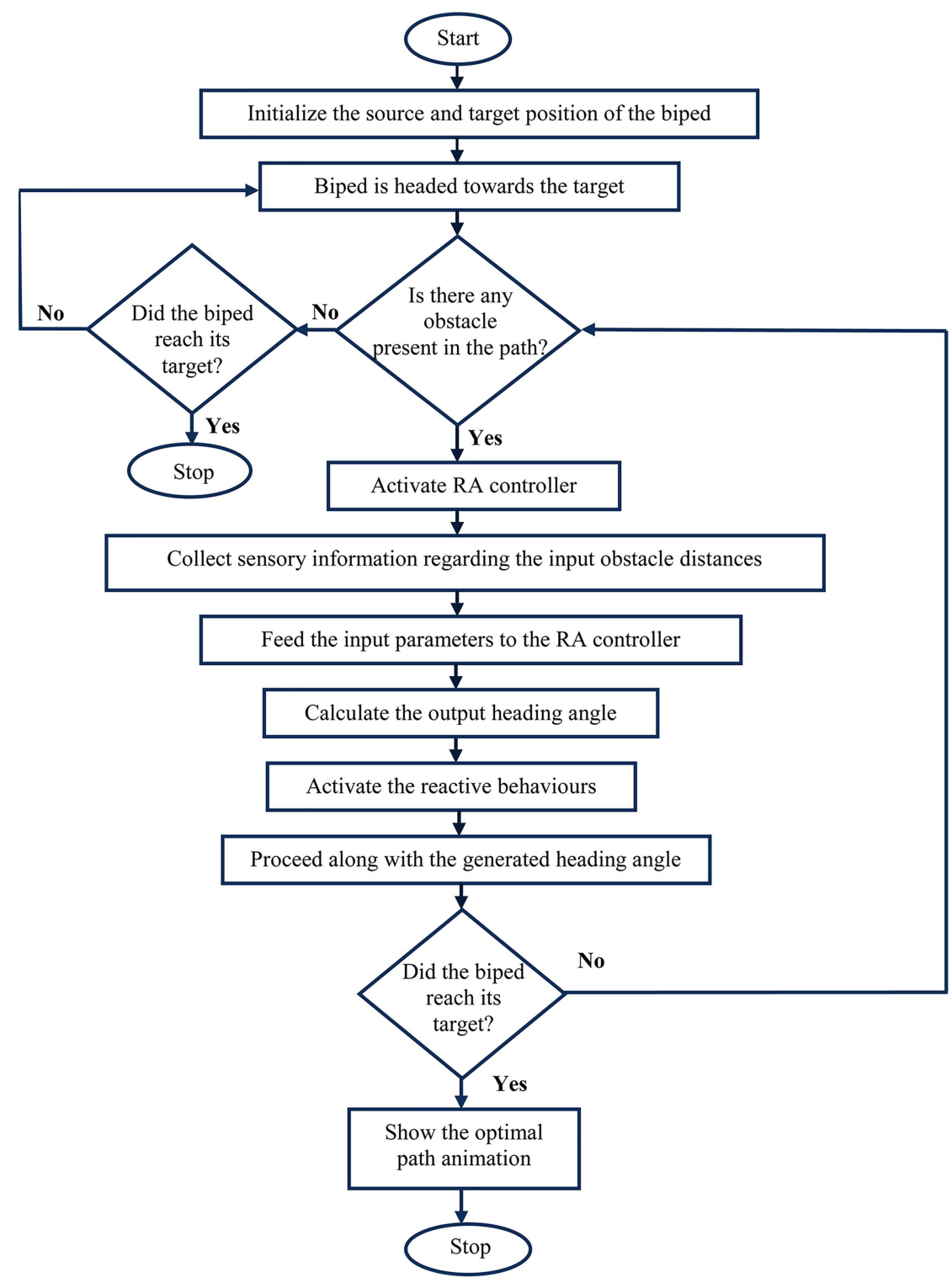

Figure 5. Flowchart of the proposed RA controller implemented on the biped.

Table 2. Comparison of path length between simulated and experimental environments.

\begin{tabular}{cccc}
\hline No. of scenario & $\begin{array}{c}\text { Path length in } \\
\text { simulation } \mathbf{( c m )}\end{array}$ & $\begin{array}{c}\text { Path length in } \\
\text { experiment } \mathbf{( c m )}\end{array}$ & \% of error \\
\hline 1 & 281.9 & 294.5 & 4.28 \\
2 & 340.5 & 359.8 & 5.36 \\
3 & 215.4 & 224.7 & 4.14 \\
Average & 279.26 & 293 & 4.59 \\
\hline
\end{tabular}

Table 3. Comparison of time consumed under simulated and experimental environments.

\begin{tabular}{cccc}
\hline No. of scenario & $\begin{array}{c}\text { Time consumed in } \\
\text { simulation (sec) }\end{array}$ & $\begin{array}{c}\text { Time consumed in } \\
\text { experiment (sec) }\end{array}$ & \% of error \\
\hline 1 & 69.56 & 73.65 & 5.55 \\
2 & 84.31 & 88.95 & 5.23 \\
3 & 52.87 & 55.23 & 4.27 \\
Average & 68.91 & 72.58 & 5.02 \\
\hline
\end{tabular}




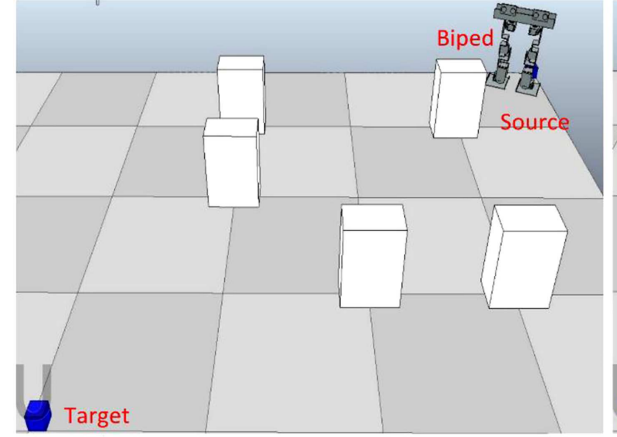

(a)

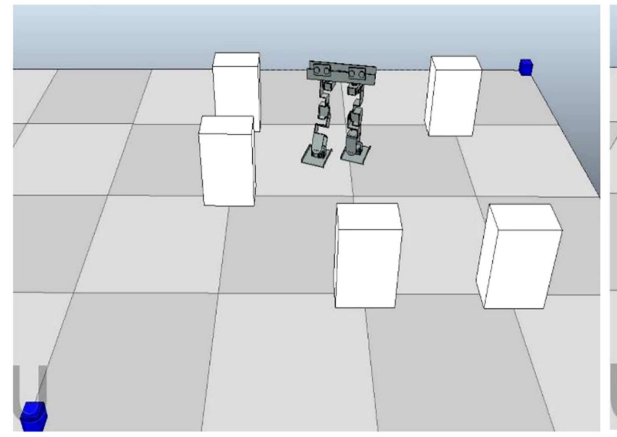

(c)

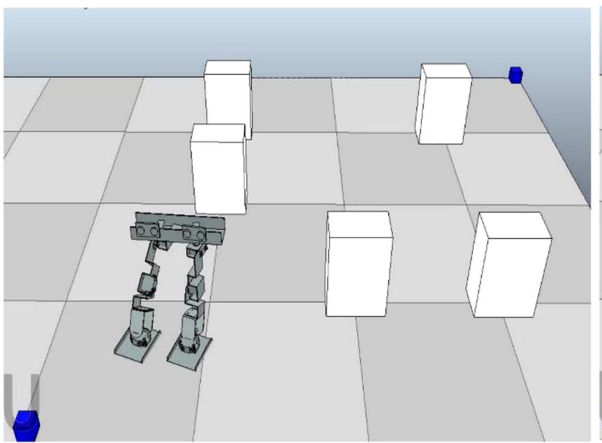

(e)

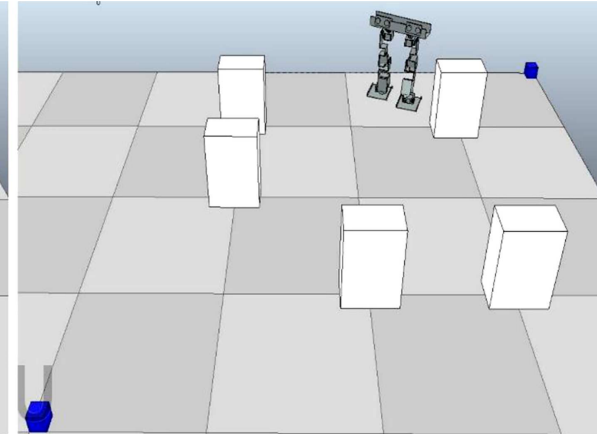

(b)

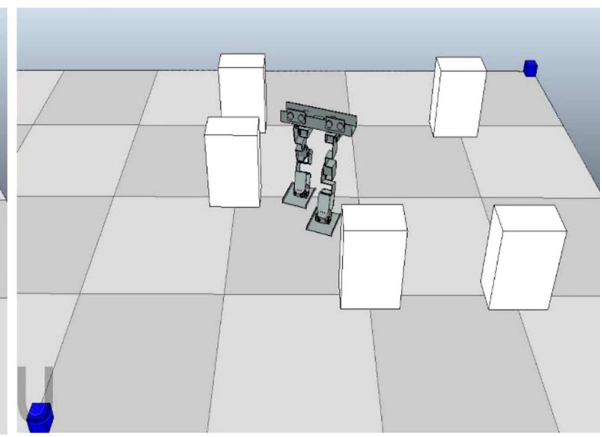

(d)

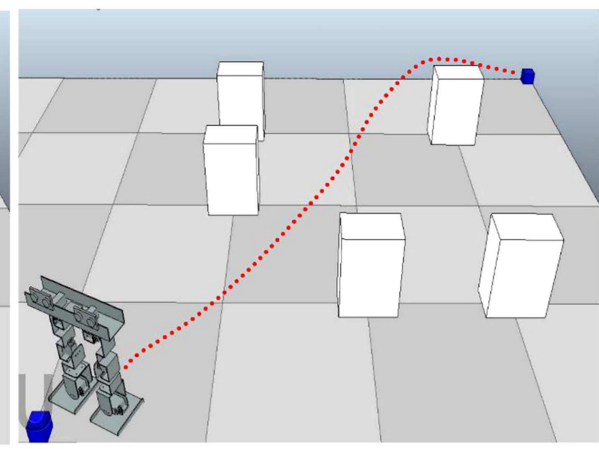

(f)

Figure 6. Simulation results obtained from the navigation of a biped robot using an RA controller.

use of regression as a navigational tool has achieved satisfactory results for the developed biped robot. However, classical approaches are always prone to lower accuracy than the soft computing techniques, and they can be easily trapped in situations such as dead end and local minima. Therefore, in further investigations, the use of various intelligence algorithms can be taken into consideration for obtaining more robust results.

\section{Conclusions}

With the development of robotics research, robots have become an integral part of human life. The increasing use of bipeds in almost all sectors of industrial automation and manufacturing has forced researchers to specifically focus on the navigation and path planning problem of biped robots. The current analysis is based on the design and implementation of a novel navigational controller for a self-fabricated biped robot considering the logic of regression analysis. After physically fabricating the biped robot, the controller of the biped was fed with the logic of regression analysis and reactive behaviors. The biped robot was tested for navigation and obstacle avoidance in a simulation platform. The results of the simulation platform were also validated through a real-time experimental setup. The biped robot was successful in avoiding all the obstacles and reaching the target position safely in both environments. The results obtained from both the platforms were closely in agreement with a minimal percentage of error. Hence, the regression controller can be used as an efficient controller for the navigation of biped robots. With a clear lack of significant research on the path planning of biped robots, the current investigation would definitely serve as a guide towards the development of more robust controllers for the same purpose. 


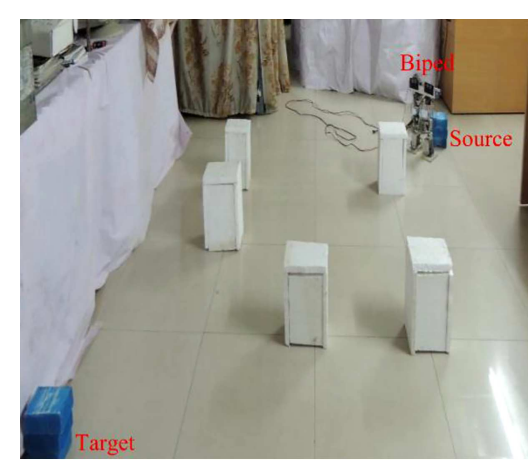

(a)

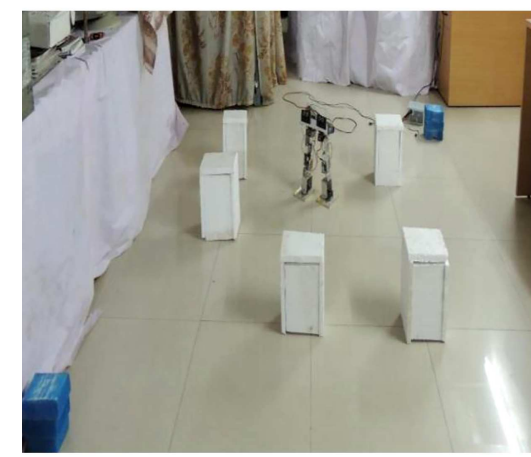

(c)

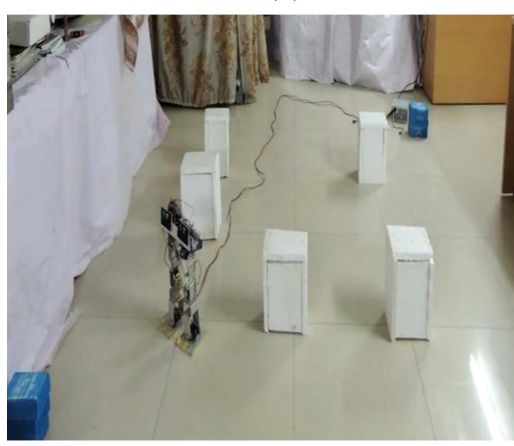

(e)

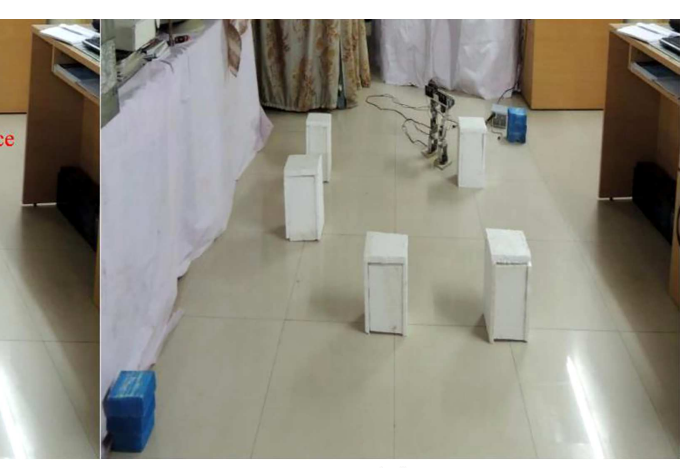

(b)

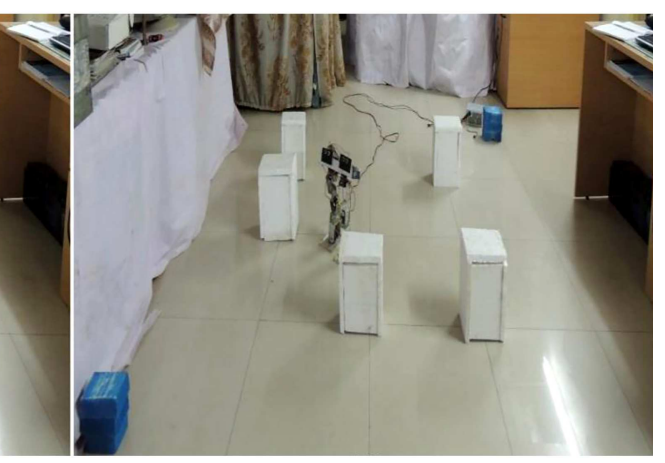

(d)

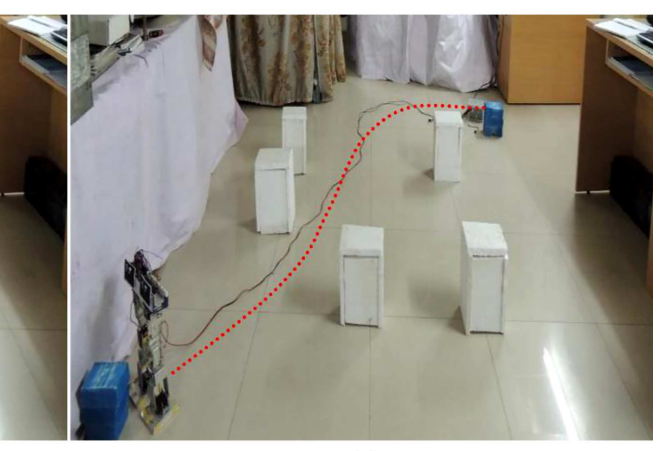

(f)

Figure 7. Experimental results obtained from the navigation of a biped robot using an RA controller.

\section{References}

1. Vukobratović, M. and Borovac, B. "Zero-moment point-thirty-five years of its life", International Journal of Humanoid Robotics, 1(01), pp. 157-173 (2004).

2. Pa, P.S. and Wu, C.M. "Design of a hexapod robot with a servo control and a man-machine interface", Robotics and Computer-Integrated Manufacturing, 28(3), pp. 351-358 (2012).

3. Liu, C., Wang, D., and Chen, Q. "Central pattern generator inspired control for adaptive walking of biped robots", IEEE Transactions on Systems, Man, and Cybernetics: Systems, 43(5), pp. 1206-1215 (2013).

4. Sahu, C., Kumar, P.B., and Parhi, D.R. "An intelligent path planning approach for humanoid robots using adaptive particle swarm optimisation", International Journal on Artificial Intelligence Tools, 27(5), p. 1850015 (2018).

5. Sahu, C., Parhi, D.R., and Kumar, P.B. "An approach to optimize the path of humanoids using adaptive ant colony optimization", Journal of Bionic Engineering, 15(4), pp. 623-635 (2018).

6. Yeon, J.S. and Park, J.H. "A fast turning method for biped robots with foot slip during single-support phase", IEEE/ASME Transactions on Mechatronics, 19(6), pp. 1847-1858 (2014).

7. Asa, K., Ishimura, K., and Wada, M. "Behavior transition between biped and quadruped walking by using bifurcation", Robotics and Autonomous Systems, 57(2), pp. 155-160 (2009).

8. Iida, F., Minekawa, Y., Rummel, J., and Seyfarth, A. "Toward a human-like biped robot with compliant legs", Robotics and Autonomous Systems, 57(2), pp. 139-144 (2009).

9. Rath, A.K., Das, H.C., Parhi, D.R., and Kumar, P.B. "Application of artificial neural network for control and navigation of humanoid robot", Journal of Mechan- 
ical Engineering and Sciences, 12(2), pp. 3529-3538 (2018).

10. Rath, A.K., Parhi, D.R., Das, H.C., Muni, M.K., and Kumar, P.B. "Analysis and use of fuzzy intelligent technique for navigation of humanoid robot in obstacle prone zone", Defence Technology, 14(6), pp. 677-682 (2018). DOI: doi.org/10.1016/j.dt.2018.03.008

11. Rath, A.K., Parhi, D.R., Das, H.C., and Kumar, P.B. "Behaviour based navigational control of humanoid robot using genetic algorithm technique in cluttered environment", Modelling, Measurement and Control A, 91(1), pp. 32-36 (2018).

12. Rushdi, K., Koop, D., and Wu, C.Q. "Experimental studies on passive dynamic bipedal walking", Robotics and Autonomous Systems, 62(4), pp. 446-455 (2014).

13. Silva, P., Santos, C.P., Matos, V., and Costa, L. "Automatic generation of biped locomotion controllers using genetic programming", Robotics and Autonomous Systems, 62(10), pp. 1531-1548 (2014).

14. Kumar, A., Kumar, P.B., and Parhi, D.R. "Intelligent navigation of humanoids in cluttered environments using regression analysis and genetic algorithm", Arabian Journal for Science and Engineering, pp. 1-24 (2018).

15. Kumar, P.B., Mohapatra, S., and Parhi, D.R. "An intelligent navigation of humanoid NAO in the light of classical approach and computational intelligence", Computer Animation and Virtual Worlds, p. e1858 (2018).

16. Kumar, P.B., Pandey, K.K., Sahu, C., Chhotray, A., and Parhi, D.R. "A hybridized RA-APSO approach for humanoid navigation", In: Nirma University International Conference on Engineering (NUiCONE), pp. 1-6 (2017).

17. Kumar, P.B., Sahu, C., and Parhi, D.R. "A hybridized regression-adaptive ant colony optimization approach for navigation of humanoids in a cluttered environment", Applied Soft Computing, 68, pp. 565585 (2018).

18. Kumar, P.B., Sahu, C., Parhi, D.R., Pandey, K.K., and Chhotray, A. "Static and dynamic path planning of humanoids using an advanced regression controller", Scientia Iranica, 26(1), pp. 375-393 (2019). DOI: 10.24200/SCI.2018.5064.1071

19. Geng, T. "Torso inclination enables faster walking in a planar biped robot with passive ankles", IEEE Transactions on Robotics, 30(3), pp. 753-758 (2014).

20. Nakamura, Y., Mori, T., Sato, M.A., and Ishii, S. "Reinforcement learning for a biped robot based on a CPG-actor-critic method", Neural Networks, 20(6), pp. 723-735 (2007).

21. Cristiano, J., Puig, D., and Garcia, M.A. "Efficient locomotion control of biped robots on unknown sloped surfaces with central pattern generators", Electronics Letters, 51(3), pp. 220-222 (2015).

22. Deepak, B.B.V.L. and Parhi, D.R. "Control of an automated mobile manipulator using artificial immune system", Journal of Experimental \& Theoretical Artificial Intelligence, 28(1-2), pp. 417-439 (2016).
23. Pandey, A. and Parhi, D.R. "Multiple mobile robots navigation and obstacle avoidance using minimum rule based ANFIS network controller in the cluttered environment", Int J Adv Robot Automation, 1(1), pp. 1-11 (2016).

24. Pandey, A., Sonkar, R.K., Pandey, K.K., and Parhi, D.R. "Path planning navigation of mobile robot with obstacles avoidance using fuzzy logic controller", In: IEEE 8th International Conference on Intelligent Systems and Control (ISCO), pp. 39-41 (2014).

25. Kajita, S., Kanehiro, F., Kaneko, K., Fujiwara, K., Harada, K., Yokoi, K., and Hirukawa, H. "Biped walking pattern generation by using preview control of zeromoment point", In: IEEE International Conference on Robotics and Automation (ICRA'03), 2, pp. 1620-1626 (2003).

26. Mirjalili, R., Yousefi-koma, A., Shirazi, F.A., and Mansouri, S. "Online path planning for SURENA III humanoid robot using model predictive control scheme", In IEEE 4th International Conference on Robotics and Mechatronics (ICROM), pp. 416-421 (2016).

27. Hwang, Y.K. and Ahuja, N. "A potential field approach to path planning", IEEE Transactions on Robotics and Automation, 8(1), pp. 23-32 (1992).

28. Atkinson, A.C. "Robust and diagnostic regression analyses", Communications in Statistics-Theory and Methods, 11(22), pp. 2559-2571 (1982).

29. Qi, N., Ma, B., Liu, X.E., Zhang, Z., and Ren, D. "A modified artificial potential field algorithm for mobile robot path planning", In: 7th World Congress on Intelligent Control and Automation, (WCICA 2008), pp. 2603-2607 (2008).

30. Asano, T., Asano, T., Guibas, L., Hershberger, J., and Imai, H. "Visibility-polygon search and Euclidean shortest paths", In IEEE 26th Annual Symposium on Foundations of Computer Science, pp. 155-164 (1985).

31. Bai, S. and Low, K.H. "Terrain evaluation and its application to path planning for walking machines", Advanced Robotics, 15(7), pp. 729-748 (2001).

32. Kala, R., Shukla, A., and Tiwari, R. "Dynamic environment robot path planning using hierarchical evolutionary algorithms", Cybernetics and Systems: An International Journal, 41(6), pp. 435-454 (2010).

33. Singh, M.K., Parhi, D.R., and Pothal, J.K. "ANFIS approach for navigation of mobile robots", In: International Conference on Advances in Recent Technologies in Communication and Computing (ARTCom'09), pp. 727-731 (2009).

34. Parhi, D.R. and Singh, M.K. "Navigational strategies of mobile robots: a review", International Journal of Automation and Control, 3(2-3), pp. 114-134 (2009).

35. Parhi, D.R. and Singh, M.K. "Real-time navigational control of mobile robots using an artificial neural network", Proceedings of the Institution of Mechanical Engineers, Part C: Journal of Mechanical Engineering Science, 223(7), pp. 1713-1725 (2009). 
36. Mohanty, P.K. and Parhi, D.R. "Optimal path planning for a mobile robot using cuckoo search algorithm", Journal of Experimental \& Theoretical Artificial Intelligence, 28(1-2), pp. 35-52 (2016).

37. Parhi, D.R. and Mohanty, P.K. "IWO-based adaptive neuro-fuzzy controller for mobile robot navigation in cluttered environments", The International Journal of Advanced Manufacturing Technology, 83(9-12), pp. 1607-1625 (2016).

38. Mohanty, P.K. and Parhi, D.R. "A new intelligent motion planning for mobile robot navigation using multiple adaptive neuro-fuzzy inference system", $A p$ plied Mathematics \& Information Sciences, 8(5), pp. $2527-2535$ (2014).

39. Sanjuan, J., Serje, D., and Pacheco, J. "Closed form solution for direct and inverse kinematics of a US-RSRPS 2-DOF parallel robot", Scientia Iranica, 25(4), pp. 2144-2154 (2018).

40. Korayem, M.H., Yousefzadeh, M., and Manteghi, S. "Tracking control and vibration reduction of flexible cable-suspended parallel robots using a robust input shaper", Scientia Iranica, Transaction B, Mechanical Engineering, 25(1), pp. 230-252 (2018).

\section{Biographies}

Priyadarshi Biplab Kumar completed his $\mathrm{PhD}$ from the Mechanical Engineering Department of National Institute of Technology, Rourkela, Odisha, India. He is currently working on the development of navigational controllers for path planning of humanoid robots. His research interests include human robot correlation, kinematics and dynamics of robots, and artificial intelligence techniques for navigational purposes.

Chinmaya Sahu completed his $\mathrm{PhD}$ from the Mechanical Engineering Department of National Institute of Technology, Rourkela, Odisha, India after completing post-graduate studies from the same institute. His research areas include humanoid robotics, path planning and navigation, and artificial intelligence techniques.

Dayal R. Parhi is serving as a Professor at the National Institute of Technology, Rourkela, Odisha, India. He has guided more than $20 \mathrm{PhD}$ dissertations. His current research interest is in various types of robots' navigation and control. Currently, he is involved in building several experimental robots for different engineering applications. 